\title{
Does delay in initiation of intravenous antibiotics correlate with wound infections in children with open fractures?
}

\author{
Chintada Anil. Kumar' ${ }^{1}$, Nathi Anil. Kumar. ${ }^{2}$ \\ ${ }^{1}$ Dr. Anil Kumar Chintada, Senior Resident, Department of Orthopaedics, Christian Medical College and Hospital, \\ Vellore, Tamilnadu, ${ }^{2}$ Dr. Anil Kumar Nathi, Assistant Professor, Department of Orthopaedics, Great Eastern Medical \\ School and Hospital, Srikakulam, Andhra Pradesh, India.
}

Corresponding Author: Dr. Anil Kumar Nathi, Flat No 202, Celesta, Aarthika Empire, D No 9-14-1, CBM Compound, VIP Road, Balajinagar, Visakhapatnam, Andhra Pradesh, 530003, India. E-mail: anilnathi@gmail.com

\begin{abstract}
Introduction: One of the common complications of open fractures is an infection which may be dependent on the time of administration of antibiotics. The study aimed to determine the prevalence of wound infection due to the delay in the interval between the initiation of intravenous (IV) antibiotics and time of paediatrics open injury. Materials and Methods: Retrospective observational study included paediatrics patients ( 0 to 16 years) with open fractures. A retrospective chart review was performed to identify patients with open fracture, who were treated between May 2012 and October 2013 at the tertiary care centre. The time between the injury and the first dose of intravenous antibiotic was calculated from transfer and hospital records. Fracture groups were stratified according to the severity of soft-tissue damage as determined with use of the Gustilo- Anderson system for the classification of open fractures. Results: Sixty patients with 84 open fractures were included in this study. Majority of 31.58\% of participants had type III B Gustilo Anderson fracture type. The proportion of grade IIIA, I, III C and II fracture was $29.82 \%, 21.05 \%, 14.04 \%$ and $3.51 \%$ respectively. $43(71.6 \%)$ received IV antibiotics within 6 hours of injury of which eight had documented wound infections. Of the 17 (28\%) patients who received IV antibiotics after 6 hours, 7 (41\%) were diagnosed with wound infections. The incidence of infection was high in higher grades of Gustilo- Anderson opens injuries. Conclusion: The immediate administration of appropriate antibiotics on presentation is crucial to minimise the risk of infection in children.
\end{abstract}

Keywords: Antibiotics, Open Fracture, Orthopaedics, Polytrauma, Paediatric

\section{Introduction}

Open fractures are defined as a break in the skin allowing the direct communication of fracture site or fracture hematoma with the external elements. In addition, all the fractures in the vicinity of any break in the skin should be treated as open fracture [1]. Open fracture makes about $10 \%$ of all paediatric-orthopaedic injuries and $25-50 \%$ of polytraumatised children have an open orthopaedic injury. Skaggs et al. have found the leg $(34 \%)$ as the most common site for open fractures and the forearm $(32 \%)$ as the second most common site [2]. In this series, the involvement of the femur and humerus were $6.7 \%$ and $6.5 \%$ respectively. The most common cause of open fractures is road traffic accidents with significant involvement of motorbikes in lower limb injuries. Fall from a height is an important cause of open injuries in the upper limb [2].

Manuscript Received: $25^{\text {th }}$ February 2019

Reviewed: $6^{\text {th }}$ March 2019

Author Corrected: $10^{\text {th }}$ March 2019

Accepted for Publication: $14^{\text {th }}$ March 2019
Open fractures in children differ from those in adults in many ways. Thicker and more active periosteum provides greater fracture stability and leads to more rapid and reliable fracture-healing in young children compared with that in older children and adults. Young children have a greater potential for periosteal bone formation and can even reconstitute bone in the face of bone loss. Common complications of open fractures are infection, neurovascular injury, delayed union, nonunion and loss of function. Infection rates in children with open fractures have been reported to be lower than those in adults with such fractures [3].

According to guidelines of Advanced Trauma Life Support (ATLS)/ Paediatric Advanced Life Support, emergent administration (within 6 hours of injury) of appropriate antibiotics is essential to decrease the risk of infection. The stabilisation of unstable fractures is usually beneficial, although children may require less 


\section{Original Research Article}

rigidity than adults. If the viability of soft tissue is in doubt, debridement should be deferred until a later operation, as the superior healing potential of young children may produce unexpected recovery [4]. Associated injuries are common with open fractures in children, and serial examinations over time often uncover these injuries [5]. This study analyses the interval between the time of open injury and initiation of intravenous (IV) antibiotics in the Emergency Department.

\section{Materials and Methods}

Study area and duration:A retrospective chart review was performed to identify patients in whom an open fracture had been treated between May 2012 and October 2013, at a tertiary care centre.

Inclusion Criteria: The study population consisted of open injuries as classified by Gustilo-Anderson classification in the age from 0 to 16 years. All open injuries including fractures, lacerations exposing bones, heel pad avulsions, vascular injuries and traumatic amputations were included in the study. Eighty consecutive patients with 104 open fractures were identified.

Exclusion criteria: Patients with injuries that resulted in death, patients who have had preliminary treatment elsewhere before coming to our institution and few patients due to lack of proper documentation (eight) were not included in the study.

Follow up: Patients were followed until there was radiographic and clinical evidence of union with absence or resolution of infection. Thus, 60 patients with 84 open fractures (and/or) extensive soft tissue injuries were included in this study.

Surgical procedure: The standard protocol at our institution was for intravenous antibiotics to be administered to all children with open fractures upon arrival in the emergency department and subsequently to be continued for at least forty-eight hours. The time between the injury and the first dose of intravenous antibiotics was calculated from transfer and hospital records. All times were rounded to the nearest hour. Fracture groups were stratified according to the severity of soft-tissue damage as determined with use of the Gustilo-Anderson system for the classification of open fractures [6]: -

Type I Wound $<1 \mathrm{~cm}$ long, clean

Type II Wound $>1 \mathrm{~cm}$ long, without extensive softtissue damage

Type III Massive soft-tissue damage, compromised vascularity, severe wound contamination, marked fracture instability

The location of the fracture was recorded. A fracture was considered to be infected if any one of three conditions was met:

(1) The patient had positive intra-operative cultures and was subsequently managed with antibiotics or additional surgical debridement,

(2) The patient did not have positive cultures but was managed with subsequent surgery or antibiotics for the treatment of infection, or

(3) The patient had a clinical diagnosis of infection, based on time taken for wound healing, serous discharge from wound. These criteria were intentionally rigid to make certain that we did not miss any cases of infection. Patients who had a single positive culture but had no clinical evidence of infection and no additional treatment were not considered to have an infection. Both deep and superficial infections were considered together. There were no cases of late infection. Pin-site infections resulting from external fixators were not considered to be wound infections.

Statistical Analysis: The data were analysed in two parts Average time delay between the time of open injury and the administration of the first dose of intravenous antibiotics in accident and emergency department of a tertiary care teaching hospital. Comparison of percentages of wound infection in children between those who received intravenous antibiotics within 6 hours and those who received after 6 hours of injury was done using Chi square test.

\section{Results}

Sixty patients with open injuries who presented to the Accident and Emergency Department of a tertiary care centre not having received preliminary treatment elsewhere were included in the study. Of these, 33 children were below 11 years of age, and 27 children were above 11 years of age. 42 children out of 60 were boys, and 18 children were girls. Six children (18\%) out of 33 under 11 years of age, and eight children (29.6\%) out of 27 over 11 years of age were diagnosed with wound infections. 43 (71.67\%) participants had $<6$ hours IV antibiotics and remaining 17 (28.33\%) participants had $>6$ hours IV antibiotics (table 1). Majority of $31.58 \%$ of participants had type III B Gustilo - Anderson fracture type. The proportion of grade III A, I, III C and II fracture was $29.82 \%, 21.05 \%, 14.04 \%$ and $3.51 \%$ respectively. (Table 1) 
Original Research Article

Table-1: Descriptive analysis of baseline characteristic in the study population $(\mathrm{N}=60)$.

\begin{tabular}{|c|c|c|}
\hline \multicolumn{2}{|c|}{ Frequency } & Percentage \\
\hline \multicolumn{3}{|c|}{ Age group } \\
\hline$<11$ years & 33 & $55 \%$ \\
\hline$>11$ years & 27 & $45 \%$ \\
\hline \multicolumn{3}{|c|}{ Gender } \\
\hline Male/Boys & 42 & $70 \%$ \\
\hline Female/girls & 18 & $30 \%$ \\
\hline \multicolumn{1}{|c|}{ Gustilo-Anderson fracture type } \\
\hline I & 12 \\
\hline II & 2 & $21.05 \%$ \\
\hline III A & 17 & $3.51 \%$ \\
\hline III B & 18 & $29.82 \%$ \\
\hline III C & 8 & $14.58 \%$ \\
\hline IV antibiotics & 43 & $71.67 \%$ \\
\hline$<6$ hours & 17 & $28.33 \%$ \\
\hline$>6$ hours & 14 & $23.33 \%$ \\
\hline Wound infection & 46 & $76.67 \%$ \\
\hline Yes & &
\end{tabular}

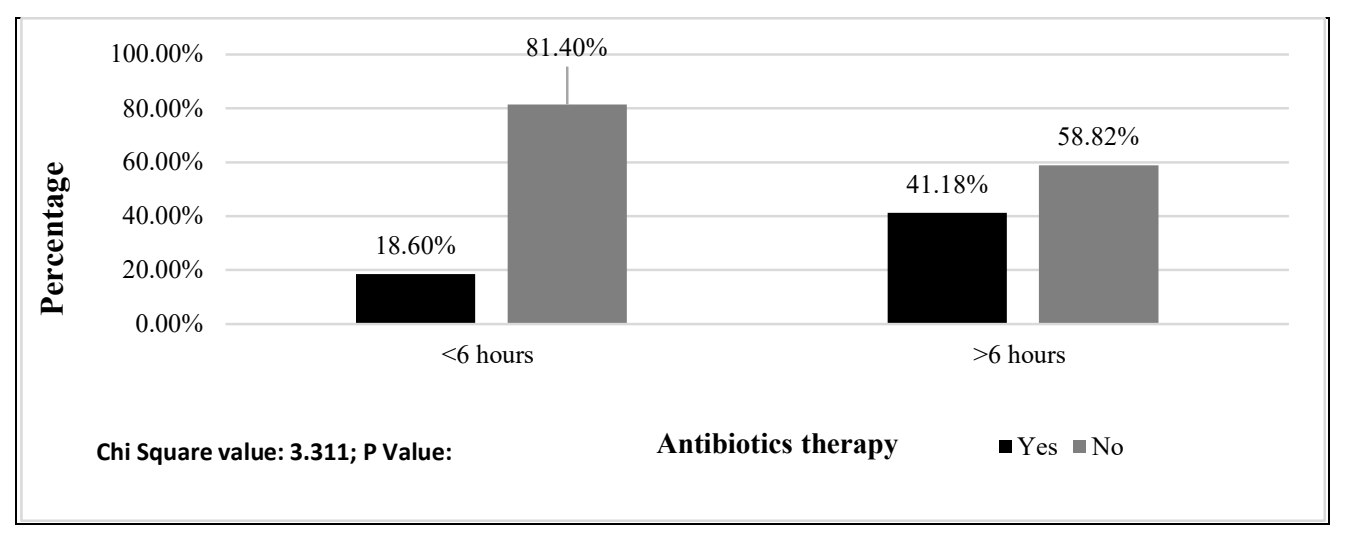

Figure 1: Comparison of incidence of infection with timing of antibiotics therapy $(\mathrm{N}=60)$

Of the 60 patients, $43(71.6 \%)$ received IV antibiotics within 6 hours of injury of which eight had documented wound infections. Of the $17(28 \%)$ patients who received IV antibiotics after 6 hours, 7 (41\%) were diagnosed with wound infections. The difference in the proportion of incidence of infection between antibiotics therapy was statistically not significant (P value 0.069) (Figure 2).

Table-2: Types of injuries and wound infection among 60 children

\begin{tabular}{|c|c|c|c|c|c|}
\hline Gustilo - Anderson Fracture TYPE & I & II & IIIA & IIIB & IIIC \\
\hline Number of children N (\%) & $12(20 \%)$ & $2(3 \%)$ & $17(28 \%)$ & $18(30 \%)$ & $8(13 \%)$ \\
\hline IV Antibiotics $<$ 6hrs & $11 / 12(91.6 \%)$ & $0 / 2(0 \%)$ & $9 / 17(53 \%)$ & $12 / 18(66.6 \%)$ & $6 / 8(75 \%)$ \\
\hline IV Antibiotics $>$ 6hrs & $1 / 12(8 \%)$ & $2 / 2(100 \%)$ & $8 / 17(47 \%)$ & $6 / 18(33 \%)$ & $2 / 8(11 \%)$ \\
\hline Number of infected cases & 0 & 0 & $2(11.7 \%)$ & $8(44 \%)$ & $5(62.5 \%)$ \\
\hline$<$ hhrs - Infected & 0 & 0 & 0 & $4(33 \%)$ & $3(50 \%)$ \\
\hline$>$ hhrs - Infected & 0 & 0 & $2(25 \%)$ & $4(67 \%)$ & $2(100 \%)$ \\
\hline
\end{tabular}

The incidence of infection was high in higher grades of Gustilo - Anderson opens injuries, which increases with an increase in the severity of the injury, especially those who received IV antibiotics after 6 hours of injury. (Table 2) 


\section{Original Research Article}

\section{Discussion}

Prompt administration of antibiotics is clearly an important way to minimise the risk of infection associated with open fractures. In our retrospective audit with 60 patients, $18.6 \%(8 / 43)$ of patients who received IV antibiotics within 6 hours of injury, had wound infection when compared to $41 \%$ (7/17) of patients who received IV antibiotics after 6 hours of injury, which is less than half the percentage of wound infection seen in the latter group. $71.6 \%$ of children received the first dose of IV antibiotic in Accident and Emergency department, within 6 hours of injury minimising the infection rate to $18.6 \%$ when compared to $41 \%$ of infection in children who received IV antibiotics after 6 hours of injury, which is recommended according to ATLS/PALS guidelines.

The incidence of infection is high in higher grades of Gustilo - Anderson opens injuries, which increases with increase in the severity of the injury, especially those who received IV antibiotics after 6 hours of injury, as shown in the above table.

Patzakis et al[5] in 1989 found in their study that the single most important factor in reducing the infection rate was the early administration of antibiotics that provide antibacterial activity against both gram-positive and gram-negative microorganisms. Similarly, Lack WD et al [7] in Multivariate analysis found antibiotics beyond 66 minutes (odds ratio, 3.78; 95\% CI, 1.16$12.31 ; \mathrm{P}=0.03$ ) independently predicted infection.

Immediate antibiotics and early coverage limited the infection rate (1 of $36,2.8 \%$ ). Swanson, T.V., et al[8] concluded that there is an increase in infection rate in the presence of wound contamination, delay in treatment greater than twenty-four hours, or systemic illness.

Merritt, in a study of seventy patients with open fractures, concluded that the time between injury and treatment in the emergency room was not correlated with infection rate, nor was the time between treatment in the emergency room and debridement in the operating room correlated with infection rate [9].

Also, Skaggs, D. L., et al[1] found in their large multicentre study, that the infection rate was $3 \%$ (twelve of 344) for fractures that had been treated within six hours after the injury, compared with $2 \%$ (four of 210) for those that had been treated at least seven hours after the injury; this difference was not significant $(\mathrm{p}=0.43)$. When the fractures were separated according to the Gustilo and Anderson classification system, there were no significant differences in the infection rate between those that had been treated within six hours after the injury and those that had been treated at least seven hours after the injury. These findings were in accordance with the current study.

Gustilo- Anderson grading system was used in the study. It provides a prognostic framework that guides treatment and facilitates communication among surgeons and clinician-scientists. Decades of research correlating the Gustilo-Anderson type with infection risk have helped refine surgical protocols, change antibiotic recommendations, and determine the appropriate timing for interventions including debridement, internal fixation, and soft tissue coverage [10-12].

It was also seen in the present study that the infection was more associated with respect to grade III B followed by grade III A injuries. Harley, B. J., et al[13] found in the multivariate regression model that time was not a significant factor in predicting either non-union or infection $(p>0.05)$. The strongest determinants for non-union were found to be the presence of infection and grade of injury $(\mathrm{p}<0.05)$. The strongest predictors for the development of a deep infection were fracture grade and a lower extremity fracture $(\mathrm{p}<0.05)$.

Noumi, T., et al [14] concluded following a multivariate analysis that Gustilo type significantly correlated with the occurrence of deep infection $(\mathrm{p}<0.05)$. Non-union occurred in 12 fractures (14.1\%). Multivariate analysis revealed that only fracture grade by AO type significantly correlated with the occurrence of nonunion $(\mathrm{p}<0.02)$.

The present study has only taken into consideration delay in antibiotic administration on the rate of infection following open fractures in children, which is one of many factors that may cause infection. The confounders were not considered in the study, and the small sample size and retrospective nature of the study reduces the generalizability of the study. The findings of the present study suggest that in children who receive early antibiotic therapy following an open fracture offers little benefit over late administration. However, a large size cohort study is needed to finalise the exact relationship between the timing of antibiotic administration and risk of infection in the case of open fractures in children. 


\section{Original Research Article}

\section{Conclusion}

Open fractures in children present special challenges. The immediate administration of appropriate antibiotics on presentation is crucial to minimise the risk of infection. Formal operative debridement of all open fractures is a time-honoured principle, although whether operative treatment within six hours rather than twentyfour hours influence the infection rate is controversial. However thorough debridement and irrigation of the wound with careful soft-tissue management are recommended which remain as the mainstay of treatment.

Contributions by authors: Dr. Chintada AK was involved in designing the study, data collection, coordinating the data analysis, writing the manuscript and approving the final draft. Dr. Nathi Ak had played supporting role in finalizing the study proposal, monitored the data quality, conducted review of literature and was involved in editing of all the drafts and approval of the final draft.

Study's addition to existing knowledge: The study flashed light on the importance of timing of administration of antibiotic and risk of infection among open fractures in children. Appropriate antibiotic course can prevent development of major risk of infections. Future studies can be planned as prospective long term followup studies.

Conflict of interest: None declared.

Funding: Nil, Permission from IRB: Yes

\section{Reference}

1. Skaggs DL, Friend L, Alman B, Chambers HG, Schmitz M, Leake B, et al. The effect of surgical delay on acute infection following 554 open fractures in children. J Bone Joint Surg Am. 2005;87(1):8-12.

2.Kay RM, Skaggs DL. Pediatric polytrauma management. J Pediatr Orthop. 2006;26(2):268-77.

3.Tscherne H, Sudkamp N. [Open fractures in children]. Z Orthop Ihre Grenzgeb. 1985;123(4):490-7.

4.Kleinman ME, de Caen AR, Chameides L, Atkins DL, Berg RA, Berg MD, et al. Pediatric basic and advanced life support: 2010 International Consensus on Cardiopulmonary Resuscitation and Emergency Cardiovascular Care Science with Treatment Recommendations. Pediatrics. 2010;126(5):e1261-318.

5. Patzakis MJ, Wilkins J. Factors influencing infection rate in open fracture wounds. Clin Orthop Relat Res. 1989 (243):36-40.

6. Gustilo RB, Anderson JT. Prevention of infection in the treatment of one thousand and twenty-five open fractures of long bones: retrospective and prospective analyses. J Bone Joint Surg Am. 1976;58(4):453-8.

7. Lack WD, Karunakar MA, Angerame MR, Seymour RB, Sims S, Kellam JF, et al. Type III open tibia fractures: immediate antibiotic prophylaxis minimizes infection. J Orthop Trauma. 2015;29(1):1-6.

8.Swanson TV, Szabo RM, Anderson DD. Open hand fractures: prognosis and classification. J Hand Surg Am. 1991;16(1):101-7.

9.Merritt K. Factors increasing the risk of infection in patients with open fractures.J Trauma.1988;28(6):823-7

10.Kim PH, Leopold SS. In brief: Gustilo-Anderson classification. [corrected]. Clin Orthop Relat Res. 2012; 470 (11):3270-4.

11.Gosselin RA, Roberts I, Gillespie WJ. Antibiotics for preventing infection in open limb fractures. Cochrane Database Syst Rev. 2004(1):CD003764.

12. Ostermann PA, Seligson D, Henry SL. Local antibiotic therapy for severe open fractures. A review of 1085 consecutive cases. J Bone Joint Surg Br. 1995; 77 (1) : 93-7.

13. Harley BJ, Beaupre LA, Jones CA, Dulai SK, Weber DW. The effect of time to definitive treatment on the rate of nonunion and infection in open fractures. J Orthop Trauma. 2002;16(7):484-90.

14. Noumi T, Yokoyama K, Ohtsuka H, Nakamura K, Itoman M. Intramedullary nailing for open fractures of the femoral shaft: evaluation of contributing factors on deep infection and nonunion using multivariate analysis. Injury. 2005;36(9):1085-93.

\section{How to cite this article?}

Chintada Anil. Kumar, Nathi Anil. Kumar. Does delay in initiation of intravenous antibiotics correlate with wound infections in children with open fractures?. Surgical Update: Int J surg Orthopedics. 2019;5(1):60-64.doi:10.17511/ ijoso.2019.i01.10. 